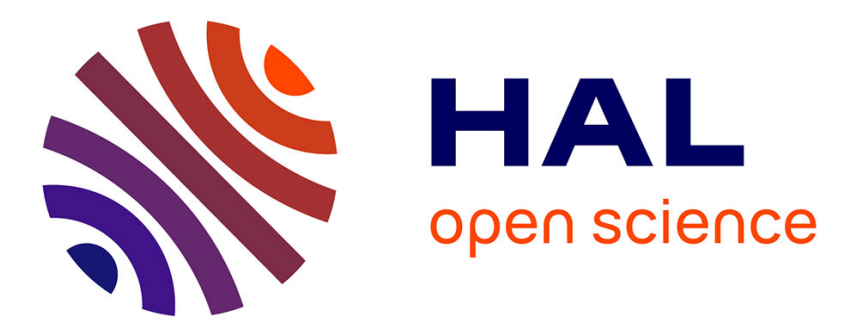

\title{
Robust Numerical Coupling of Pressure and Pressureless Gas Dynamics Equations for Eulerian Spray DNS and LES
}

\author{
Matthieu Boileau, Christophe Chalons, Marc Massot
}

\section{- To cite this version: \\ Matthieu Boileau, Christophe Chalons, Marc Massot. Robust Numerical Coupling of Pressure and Pressureless Gas Dynamics Equations for Eulerian Spray DNS and LES. SIAM Journal on Scientific Computing, 2015, 37 (1), pp.79-102. 10.1137/130945740 . hal-00906220v2}

\section{HAL Id: hal-00906220 \\ https://hal.science/hal-00906220v2}

Submitted on 27 Feb 2015

HAL is a multi-disciplinary open access archive for the deposit and dissemination of scientific research documents, whether they are published or not. The documents may come from teaching and research institutions in France or abroad, or from public or private research centers.
L'archive ouverte pluridisciplinaire HAL, est destinée au dépôt et à la diffusion de documents scientifiques de niveau recherche, publiés ou non, émanant des établissements d'enseignement et de recherche français ou étrangers, des laboratoires publics ou privés. 


\title{
ROBUST NUMERICAL COUPLING OF PRESSURE AND PRESSURELESS GAS DYNAMICS EQUATIONS FOR EULERIAN SPRAY DNS AND LES*
}

\author{
M. BOILEAU ${ }^{\dagger}$, C. CHALONS ${ }^{\ddagger}$, AND M. MASSOT $^{\dagger}$
}

\begin{abstract}
Large eddy simulation (LES) and direct numerical simulation (DNS) of polydisperse evaporating sprays with Eulerian models are very promising tools for high performance computing of combustion applications since they are able to predict the turbulent dispersion and evaporation. However, the spray system of conservation equations has a convective part which is similar either to gas dynamics Euler equations with a real gas type state law or to the pressureless gas dynamics (PGD), depending on the local flow regime and droplet Stokes number; so they usually involve singularities due to model closure assumptions and require dedicated numerical schemes. The present contribution introduces a new generation of numerical methods based on relaxation schemes which are able to treat both PGD and general gas dynamics as well as to cope in a robust manner with vacuum zones and natural singularities of the resulting system of conservation equations. The approach relies on the coupling between a relaxed model for PGD and a relaxed model for gas dynamics using an energy threshold. The proposed hybrid relaxation scheme and algorithms are validated through comparisons with analytical solutions and other numerical strategies on one-dimensional (1D) and two-dimensional (2D) configurations. They exhibit a very robust behavior and are a very promising candidate for more complex applications since they provide solutions to key numerical issues of the actual Eulerian spray DNS and LES models. Though the energy is considered here as isotropic, the method can be extended to nonequilibrium gas dynamics to describe the spray dynamics with higher accuracy.
\end{abstract}

Key words. relaxation schemes, spray equation, multifluid models, large eddy simulation

AMS subject classifications. 35L60, 35L65, 35L67, 65Z05, 65M08, 76T15, 76F65

DOI. $10.1137 / 130945740$

1. Introduction. Many industrial devices involve turbulent combustion of a liquid fuel. The transportation sector, rocket, aircraft, or car engines are almost exclusively based on storage and injection of a liquid phase, which is sprayed into a combustion chamber. It is of primary importance to understand and control the physical process as a whole, from the injection into the chamber up to the combustion phenomena. Numerical simulation is now a standard industrial tool to optimize the turbulent combustion process in such devices [11]. Thanks to large eddy simulation (LES), unsteady phenomena such as jet ignition [20] and combustion instabilities [34, 33] can now be accurately predicted in simplified configurations where purely gaseous flames are encountered. Nevertheless, the liquid fuel injection needs special attention in order to properly predict the combustion regimes. It consists in two parts. The first is related to the atomization process near the injector and requires dedicated models and methods. The second part is related to the spray dynamics once the liquid has reached the structure of a polydisperse cloud of droplets; some promising advances

* Submitted to the journal's Computational Methods in Science and Engineering section November 18, 2013; accepted for publication (in revised form) December 1, 2014; published electronically February 12, 2015.

http://www.siam.org/journals/sisc/37-1/94574.html

${ }^{\dagger}$ Laboratoire EM2C - CNRS UPR 288 and Fédération de Mathématiques - CNRS FR 3487, École Centrale Paris, 92295 Châtenay-Malabry, France (matthieu.boileau@ecp.fr, marc.massot@ecp.fr).

${ }^{\ddagger}$ Laboratoire de Mathématiques de Versailles, UMR CNRS 8100, Université de Versailles SaintQuentin-en-Yvelines, UFR des Sciences, bâtiment Fermat, 45 avenue des Etats-Unis, 78035 Versailles cedex, France and Fédération de Mathématiques - CNRS FR 3487, École Centrale Paris, 92295 Châtenay-Malabry, France (christophe.chalons@uvsq.fr). 
have been performed in the field of spray combustion in real devices [3, 4, 40, 39]. However, the reliable prediction of such complex two-phase reacting flows requires further work in the modeling of the triple spray/turbulence/combustion interaction. In particular, the description of the turbulent spray dispersion remains a challenging issue. Spray models have a common basis at the mesoscopic level under the form of a number density function (NDF) satisfying a Boltzmann-type equation, the socalled Williams equation [41]. The internal variables characterizing one droplet are the size, the velocity, and the temperature, so that the total phase space is usually high-dimensional. Such a transport equation describes the evolution of the NDF of the spray due to convection, heating, evaporation, and drag force from the gaseous phase and droplet-droplet interactions. Different strategies can be used to solve the dispersed phase dynamics. A first choice is to approximate the NDF by a sample of discrete numerical parcels of particles through a Lagrangian-Monte Carlo approach (see [28]). It is called the direct simulation Monte Carlo method (DSMC) in [2] and is generally considered to be the most accurate for solving the Williams equation; it is especially suited for direct numerical simulation (DNS) since it does not introduce any numerical diffusion, the particle trajectories being exactly solved. This approach has been widely used and has been shown to be efficient in numerous cases. Its main drawback is the delicate coupling between the Lagrangian description of the dispersed phase and the Eulerian description of the gaseous phase. Moreover, from a computational point of view, a Lagrangian solver is difficult to efficiently parallelize using the domain decomposition for the gas solver. This is particularly true in massively parallel calculations where only a few parallel blocks may contain most of the Lagrangian particles and need dedicated algorithms as in [14]. Finally, unsteady computations of polydisperse sprays require a large number of parcels in each Eulerian cell, leading to large memory needs and high CPU costs. As a consequence, as long as it is able to describe the essential feature of polydispersity, an Eulerian formulation for the dispersed phase is more attractive for massively parallel simulations of industrial configurations, even if in both Eulerian and Lagrangian approaches the grid of the gaseous carrier phase will impose a length scale below which smaller scales are not resolved.

Based on [16] and [22], a multidimensional Eulerian multifluid solver capable of describing the polydispersity of a spray in size and the associated size-conditioned dynamics has been developed in [10]. This approach relies on the derivation of a semikinetic model from the Williams equation using a moment method for velocity conditioned by droplet size while keeping the continuous size distribution function. The key issue is in the velocity moment closure for which two strategies exist. The first, based on the quadrature method (see [18] and references therein), has been developed to capture strongly nonequilibrium velocity distributions and will not be treated in the present contribution. The second, adopted in the present paper, is based on equilibrium velocity distributions either with zero dispersion around the mean in the framework of DNS such as in $[10,13]$ and references therein or with nonzero dispersion in the framework of ensemble averages and modeling nonresolved scales such as in $[24,26,40]$.

In the context of spray dynamics, a zero pressure assumption means that the probability density function (PDF) of particle velocity is a Dirac in the velocity space, i.e., that no dispersion in the local instantaneous particle velocity is considered. For this assumption to be true, the relaxation time of particles must not exceed the timescale of the fluid turbulence. Otherwise, the effect of trajectory crossing due to 
higher-inertia particles induces a random-uncorrelated component of particle motion that must be taken into account; such trajectory crossings are usually described with difficulty by Eulerian models, whereas Lagrangian approaches naturally capture them. In [35] and [19] the authors proposed a formalism that accounts for this randomuncorrelated motion in the context of DNS. This approach has been extended for LES in [27] and [31, 32]. The authors used a spatial filtering of Kaufmann's system of Eulerian conservation equations and proposed a model for the resulting particulate subgrid stresses. Another way to use the LES concept for the spray equations is to apply the spatial filtering directly to the Williams equation for the PDF of particle velocity (see [29] and [43]). The resulting kinetic equation for the filtered PDF has the same form as the statistical PDF equation initially derived by [30] in the context of Reynolds-averaged Navier-Stokes (RANS) and used in [26]. Whatever the approach for turbulence modeling (DNS, LES, or RANS) and the level of corresponding filtering (on the kinetic equation or on the moment equations at the semikinetic level), the local velocity dispersion of particles introduces stress, and more specifically a pressure-like term, in the spray conservation equations. Finally, the Eulerian equations for inertial particles dynamics are similar to the gas dynamics equations. In particular, they include a real gas type state law which can eventually degenerate in some parts of the flow to a zero pressure term leading to the peculiar pressureless gas dynamics (PGD). Let us emphasize that the size distribution function is then discretized using a finite volume approach in the size phase space that yields conservation equations for mass and momentum (and eventually other properties such as enthalpy) of droplets in fixed size intervals which have the same mathematical structure. In the present paper, we will consider a monodisperse spray so that the semikinetic model is sufficient, keeping in mind that all the developed tools can be easily extended to polydispersity in the framework of the multifluid method $[10,40]$.

The main difficulty of the resulting system of conservation equations is related to transport in physical space, that is, the convective part of the system, which is either hyperbolic or weakly hyperbolic, and thus leads to singularity formation. In the framework of the PGD system, the problem has been solved in [10] by using a numerical strategy based on the kinetic scheme of Bouchut, Jin, and Li [6] (called the BJL scheme in what follows) which leads to a second-order method in space and time with very limited diffusion. This numerical scheme makes it possible to accurately capture the delta-shocks in density and vacuum states which naturally emerge from the weakly hyperbolic system. However, this strategy cannot be extended in a natural manner to gas dynamics either with the perfect gas law or with real gas state laws.

Therefore, the numerical method we are looking for must have the ability (1) to handle an Euler-type system of equations in regions of high Stokes number or in regions where subgrid scales induce significant pressure effects, (2) to degenerate to the PGD system in regions of Stokes number below the critical value for particular trajectory crossing or in regions where the subgrid scales do not play any role in particle velocity dispersion, and (3) to treat exact vacuum regions for both pressureless and full gas dynamics systems in various regions of the flow. Moreover, this method must feature the same properties of robustness with singularities and vacuum treatment as the BJL kinetic scheme for PGD proposed in $[10,25]$. Finally, since the pressure law can bear some real gas effects, the numerical method has to handle such cases while keeping a high level of accuracy as required by the DNS/LES approach.

In that context, the purpose of the present paper is to introduce a novel numerical method based on relaxation schemes which has the ability to match all the previous 
requirements. Relaxation methods, introduced in [17], and further developed in [36] and [9], have a common basis: they introduce auxiliary variables in the framework of Godunov schemes in order to treat more easily the strong nonlinearity due to the treatment of pressure and state law. They avoid using complex nonlinear Riemann solvers or their approximated versions which can have a very high computational cost with nonstandard pressure laws. The nonlinearity treatment is replaced by a splittinglike strategy in the framework of a linear or linearly degenerate version of the transport step along with a strong relaxation step related to a singular perturbation parameter. A large literature on the subject has shown the impact of such seminal ideas $[1,5,7,8]$ (among others).

In this contribution, we conduct three new steps: (1) Extending the work of [1], we propose a scheme for PGD based on successive energy and pressure relaxation which can deal with vacuum; (2) based on this new scheme, we introduce a hybrid numerical method which can treat both regions with and without pressure and still remain accurate and robust; and (3) we finally prove the potential of these schemes by comparing them on several tough test-cases to standard approaches in both onedimensional (1D) and two-dimensional (2D) configurations. Since relaxation methods are able to treat arbitrary state law, we only provide the schemes in the framework of the ideal gas law; additionally we focus on the purely convective part of the system of conservation laws and do not treat the potential stress tensors which can be handled by standard schemes. The paper is organized as follows. The first section describes how the Eulerian description of turbulent spray dynamics leads to a gas dynamicstype system of conservation equations. The hybrid relaxation scheme is derived in the second section. Finally, results for relevant test-cases, in one and two dimensions and pressureless/pressure/hybrid configurations, are presented and discussed.

\section{Eulerian modeling of turbulent spray dynamics.}

2.1. Conservation equations on moments of the particle number density function. At the mesoscopic level, spray models have a common basis called the kinetic model by analogy with kinetic theory of gases. The spray is described as a statistical cloud of point particles experiencing exchanges of mass, momentum, and heat with the carrier phase. This kinetic model is described by a Boltzmann-type equation (2.1) for the number density function (NDF) $f$ of the spray, where $f(t, \mathbf{x}, \mathbf{u}) \mathrm{d} \mathbf{x} \mathrm{d} \mathbf{u}$ denotes the probable number of particles at time $t$, in a volume of size $\mathrm{d} \mathbf{x}$ around $\mathbf{x}$, with a velocity in a du-neighborhood of $\mathbf{u}$. As mentioned in the introduction, other physical properties like the particle size and temperature can be introduced in the NDF for a finer description of the spray in the framework of the multifluid model introduced in [22] and for which references are to be found in [10]. For the sake of simplicity, constant particle size (monodisperse spray) and temperature are considered here, and so these variables will not appear in the equations.

The evolution of the spray NDF is given by the Williams transport equation [41],

$$
\partial_{t} f+\mathbf{u} \cdot \partial_{\mathbf{x}} f+\partial_{\mathbf{u}} \cdot(\mathbf{F} f)=0
$$

where $\mathbf{F}$ is the drag force due to the velocity difference with the gaseous phase and given by the Stokes law,

$$
\mathbf{F}(t, \mathbf{x}, \mathbf{u})=\frac{\mathbf{U}(t, \mathbf{x})-\mathbf{u}}{\tau_{p}} \quad \text { with } \tau_{p}=\frac{\rho_{l} d^{2}}{18 \mu_{g}},
$$

where $\mathbf{U}$ is the gas velocity at the particle location, $\tau_{p}$ is the particle relaxation time, 
$\mu_{g}$ is the gas viscosity, and $\rho_{l}$ and $d$ are the mass density and diameter of the particle, respectively.

The first possibility is to write conservation equations for the zero- and first-order moments with respect to the velocity variable at a given time $t$ and position $\mathbf{x}$ :

$$
\begin{aligned}
n(t, \mathbf{x}) & =\int f(t, \mathbf{x}) \mathrm{d} \mathbf{u}, \\
n(t, \mathbf{x}) \overline{\mathbf{u}}(t, \mathbf{x}) & =\int \mathbf{u} f(t, \mathbf{x}) \mathrm{d} \mathbf{u},
\end{aligned}
$$

where $n$ is the particle density and $\overline{\mathbf{u}}$ is the particle mean velocity. At this stage, there are two different ways of deriving the conservation equations for these two moments according to the value of the particle Stokes number St, defined by St $=\tau_{p} / \tau_{K}$, where $\tau_{K}$ is the Kolmogorov time microscale.

2.2. Pressureless gas system. For low Stokes numbers, particles have a low inertia and do not experience any trajectory crossings. Accordingly, the velocity dispersion around the averaged velocity $\overline{\mathbf{u}}(t, \mathbf{x}, S)$ is assumed to be zero in each directionthe spray is called monokinetic - and the NDF writes as

$$
f(t, \mathbf{x}, \mathbf{u})=n(t, \mathbf{x}) \delta(\mathbf{u}-\overline{\mathbf{u}}(t, \mathbf{x})) .
$$

Such an assumption leads to a closed system of conservation equations given by two partial differential equations in the variables $n(t, \mathbf{x})$ and $\overline{\mathbf{u}}(t, \mathbf{x})$ which express the conservation of the number density of droplets and their momentum, respectively:

$$
\left\{\begin{aligned}
\partial_{t} n+\partial_{\mathbf{x}} \cdot(n \overline{\mathbf{u}}) & =0, \\
\partial_{t}(n \overline{\mathbf{u}})+\partial_{\mathbf{x}} \cdot(n \overline{\mathbf{u}} \otimes \overline{\mathbf{u}}) & =n \overline{\mathbf{F}},
\end{aligned}\right.
$$

where the Stokes drag $\overline{\mathbf{F}}$ is taken at $\mathbf{u}=\overline{\mathbf{u}}$. Equation (2.6) is similar to the PGD system with an additional velocity relaxation source term.

2.3. Gas dynamics system. As pointed out in the introduction, the monokinetic assumption is not verified for larger Stokes numbers, i.e. for particle relaxation times greater than the Kolmogorov timescale, where the effects of particles trajectory crossings require additional higher-order moment modeling. In particular, these crossings are expected to reduce the particle segregation induced by inertia effects. A way to account for the uncorrelated motion of inertial particles is to use the mesoscopic formalism proposed by [12], starting from the following decomposition: $\mathbf{u}=\overline{\mathbf{u}}(t, \mathbf{x})+\delta \mathbf{u}$, where $\delta \mathbf{u}$ is called the random uncorrelated component of the particle velocity. Using this decomposition, one can introduce the particle uncorrelated energy $\delta \theta$ which traduces a distribution of particle velocity at a given location of the spray. This velocity distribution is generally the consequence of the turbulence of the carrier flow, and the uncorrelated energy may be related to the turbulent energy [12]. System (2.6), obtained for a monokinetic spray, now becomes (see [19])

$$
\left\{\begin{aligned}
\partial_{t} n+\partial_{\mathbf{x}} \cdot(n \overline{\mathbf{u}}) & =0, \\
\partial_{t}(n \overline{\mathbf{u}})+\partial_{\mathbf{x}} \cdot(n \overline{\mathbf{u}} \otimes \overline{\mathbf{u}}+\mathcal{P}) & =n \overline{\mathbf{F}}+\partial_{\mathbf{x}} \delta \tau, \\
\partial_{t}(n \mathcal{E})+\partial_{\mathbf{x}} \cdot(n \mathcal{E} \overline{\mathbf{u}}+\mathcal{P} \overline{\mathbf{u}}) & =n \overline{\mathbf{F}} \cdot \overline{\mathbf{u}}-2 \frac{n}{\tau_{p}} \delta \theta+\mathcal{T}_{\delta \theta},
\end{aligned}\right.
$$


where the total energy reads $\mathcal{E}=\overline{\mathbf{u}} \cdot \overline{\mathbf{u}} / 2+\delta \theta$, where the random uncorrelated energy $\delta \theta$ is defined as half the trace of the random uncorrelated stress tensor and where $\mathcal{P}$ is called the random uncorrelated pressure, which is linked to the random uncorrelated energy through the following equation of state:

$$
\mathcal{P}=\frac{2}{3} n \delta \theta
$$

In system $(2.7), \delta \tau$ is the deviatoric part of the random uncorrelated motion tensor, and it can be modeled by a viscosity assumption. $\mathcal{T}_{\delta \theta}$ gathers the effect of the transport of uncorrelated energy by the uncorrelated motion and the effect of the random uncorrelated stress tensor.

These equations correspond to the case where the gas flow is entirely resolved and no modeling of the gas turbulence is used (DNS approach). In the context of statistical (RANS) filtering [10, 26, 30, 42] or LES filtering [27, 29, 31, 43], the pressure law becomes more complicated than (2.8), involving contributions from turbulent or subgrid motion, respectively. The modeled scales involve real gas effects through a modification of the state law, as well as source terms of random uncorrelated energy in the right-hand side (RHS) of system (2.7).

The simplified general form of the system of conservation equations finally considered in the following is then system (2.7) with $\delta \tau=0$ and the additional term $\mathcal{T}_{\delta \theta}=0$ but with a potential source term: $\frac{2 n}{\tau} \delta \theta$ is replaced by $\frac{2 n}{\tau}\left(\delta \theta-\varepsilon_{t}\right)$, where $\varepsilon_{t}$ is the energy source term due to subgrid turbulence agitation. From a numerical point of view, we thus isolate the difficulties of solving system (2.7). These difficulties require a numerical method for highly compressible flows. The additional source terms and second-order derivatives usually do not lead to numerical difficulties and can be treated through operator splitting, whereas the main difficulties arise from the convective first-order part involving the pressure effects. Therefore, in the next subsection, we will focus on the left-hand side (LHS) and will temporarily forget the RHS in order to build the numerical schemes. In the following, we focus on the perfect gas state law, but relaxation methods can be easily extended to any state law with real gas effects usually encountered in LES. Finally, we also need to be able to treat cases where the random uncorrelated energy can be zero and the previous system degenerates toward the PGD.

3. A hybrid relaxation scheme for gas/pressureless gas dynamics problems. Our objective in this section is to describe a global numerical strategy in one dimension, able to deal with both gas dynamics and PGD at the same time, and to handle vacuum. It is based on the concept of relaxation approximation for systems of conservation laws. The basic idea is to propose an enlarged system with a stiff relaxation source term, the solutions of which are expected to converge to the solutions of the initial system in the asymptotic limit. In the following, and for the sake of clarity, the notation of the previous section is abandoned and replaced by the more usual notation for hyperbolic systems of conservation laws. We do not take into account the drag force in this section.

3.1. Toward a unified treatment of gas and pressureless gas dynamics. We first propose writing the pressureless gas system (2.6) (without drag terms) under the equivalent form

$$
\left\{\begin{array}{l}
\partial_{t} \rho+\partial_{x}(\rho u)=0 \\
\partial_{t}(\rho u)+\partial_{x}\left(\rho u^{2}+p\right)=0
\end{array}\right.
$$


with $p=0$. Then, following the general idea of [9], we propose approximating the solutions of this system by those of the energy relaxation system

$$
\left\{\begin{array}{l}
\partial_{t} \rho+\partial_{x}(\rho u)=0 \\
\partial_{t}(\rho u)+\partial_{x}\left(\rho u^{2}+p\right)=0, \\
\partial_{t}(\rho E)+\partial_{x}(\rho E u+p u)=-\lambda \rho \varepsilon
\end{array}\right.
$$

where the so-called relaxation internal and total energies are related by $E=\varepsilon+\frac{1}{2} u^{2}$. Importantly, the pressure $p$ here no longer equals zero but obeys, for instance, a perfect gas equation of state,

$$
\rho \varepsilon=\frac{p}{\gamma-1}
$$

At least formally, we observe from the last equation in (3.2) that the relaxation internal energy $\rho \varepsilon$ tends to zero as the relaxation parameter $\lambda>0$ goes to infinity. By (3.3), the solutions of the relaxation system (3.2) are thus expected to provide a good approximation of the solutions of the PGD for large values of $\lambda$. Note that if we define the temperature $T$ and the mathematical entropy $S$ according to the second principle of thermodynamics $-T d S=d \varepsilon-p d \tau, \tau=1 / \rho$, easy calculations lead to the expected entropy inequality $\partial_{t}(\rho S)+\partial_{x}(\rho S u)=-\lambda \rho \varepsilon \leq 0$. It is important to note that the zero internal energy equilibrium manifold, which is also the zero pressure manifold in the limit of infinite $\lambda$, is stable in the sense that for initial data with zero pressure, the dynamics naturally remain with zero pressure.

The numerical procedure we are going to propose in order to approximate the solutions of the PGD system (3.1) is very classical in the context of relaxation approximations. It is based on an operator splitting for (3.2) and is made of two steps that we now briefly describe.

First step. We solve the convective part of the model:

$$
\left\{\begin{array}{l}
\partial_{t} \rho+\partial_{x}(\rho u)=0 \\
\partial_{t}(\rho u)+\partial_{x}\left(\rho u^{2}+p\right)=0 \\
\partial_{t}(\rho E)+\partial_{x}(\rho E u+p u)=0
\end{array}\right.
$$

which is nothing but the classical gas dynamics system. We will use the condensed form

$$
\partial_{t} \mathcal{U}+\partial_{x} \mathcal{F}(\mathcal{U})=0
$$

for (3.4) with clear definitions for $\mathcal{U}$ and $\mathcal{F}(\mathcal{U})$.

Second step. In the second step, the contribution of the stiff relaxation source term is accounted for by solving the ODE system

$$
\left\{\begin{array} { l } 
{ \partial _ { t } \rho = 0 , } \\
{ \partial _ { t } ( \rho u ) = 0 , } \\
{ \partial _ { t } ( \rho E ) = - \lambda \rho \varepsilon }
\end{array} \Longleftrightarrow \left\{\begin{array}{l}
\partial_{t} \rho=0, \\
\partial_{t}(\rho u)=0, \\
\partial_{t} \varepsilon=-\lambda \varepsilon
\end{array}\right.\right.
$$

in the asymptotic regime $\lambda \rightarrow \infty$. This clearly amounts to keeping $\rho$ and $\rho u$ unchanged and to setting $\varepsilon=0$, that is, $\rho E=\frac{1}{2} \rho u^{2}$ and $p=0$.

3.2. A pressure relaxation model for gas dynamics. In this paragraph, we propose a pressure relaxation system in order to approximate the solutions of the gas dynamics system (3.4). Motivated by the seminal work of [17] and [36], we relax the 
nonlinearities associated with the pressure law $p$ only and retain the others for the sake of accuracy. With this in mind and similarly to $[1,5,7,8]$ (among others), we introduce the following nonlinear first-order system with singular perturbation:

$$
\left\{\begin{array}{l}
\partial_{t} \rho+\partial_{x}(\rho u)=0 \\
\partial_{t}(\rho u)+\partial_{x}\left(\rho u^{2}+\Pi\right)=0 \\
\partial_{t}(\rho E)+\partial_{x}(\rho E u+\Pi u)=0 \\
\partial_{t}(\rho \Pi)+\partial_{x}\left(\rho \Pi u+a^{2} u\right)=\mu \rho(p-\Pi),
\end{array}\right.
$$

which we write for brevity as $\partial_{t} \mathcal{V}+\partial_{x} \mathcal{G}(\mathcal{V})=\mu \mathcal{R}(\mathcal{V})$. As $\mu$ goes to infinity, we observe at least formally that the relaxation pressure $\Pi$ tends to $p$ so that the equilibrium system (3.4) is recovered in this asymptotic regime. The additional equation associated with $\Pi$ is easily seen to be equivalent to $\partial_{t} \Pi+u \partial_{x} \Pi+\frac{a^{2}}{\rho} \partial_{x} u=\mu(p-\Pi)$. This equation is then very similar to that associated with the exact pressure $p$ given by $\partial_{t} p+u \partial_{x} p+\rho c^{2} \partial_{x} u=0$. The choice of the parameter $a>0$ is crucial for the stability of the relaxation procedure and is determined by the so-called subcharacteristic condition $a>\rho c$, where $c$ denotes the sound speed.

The first-order system extracted from (3.7) is hyperbolic and admits the three eigenvalues $\lambda_{1}=u-a / \rho, \lambda_{2}=u, \lambda_{3}=u+a / \rho$, with second-order multiplicity for $\lambda_{2}$. We note that $\lambda_{1}$ and $\lambda_{3}$ approximate the characteristic speeds $u-c$ and $u+c$ of (3.4). Importantly, these eigenvalues are now associated with linearly degenerate characteristic fields. This implies that the Riemann problem associated with (3.7) (with $\mu=0$ ) can be explicitly solved, unlike the one associated with (3.4). Riemann solutions being the key ingredient to devise Godunov-type methods, this mathematical property justifies the introduction of the relaxation model (3.7).

Here again, the proposed numerical procedure to approximate the solutions of the gas dynamics system (3.4) is based on a classical operator splitting for (3.7) (see again $[1,5,7,8,17,36]$ among others) and is made up of two steps.

First step. We solve the convective part of the pressure relaxation model taking $\mu=0$ in (3.7):

$$
\left\{\begin{array}{l}
\partial_{t} \rho+\partial_{x}(\rho u)=0 \\
\partial_{t}(\rho u)+\partial_{x}\left(\rho u^{2}+\Pi\right)=0 \\
\partial_{t}(\rho E)+\partial_{x}(\rho E u+\Pi u)=0 \\
\partial_{t}(\rho \Pi)+\partial_{x}\left(\rho \Pi u+a^{2} u\right)=0
\end{array}\right.
$$

or, equivalently,

$$
\partial_{t} \mathcal{V}+\partial_{x} \mathcal{G}(\mathcal{V})=0
$$

In practice, we will use in this step a Godunov method based on the exact Riemann solution of (3.8).

Second step. We then solve

$$
\left\{\begin{array}{l}
\partial_{t} \rho=0 \\
\partial_{t}(\rho u)=0 \\
\partial_{t}(\rho E)=0 \\
\partial_{t}(\rho \Pi)=\mu \rho(p-\Pi)
\end{array}\right.
$$

in the asymptotic regime $\mu \rightarrow \infty$. The conservative variables $\rho, \rho u$, and $\rho E$ are thus constant, while $\Pi$ is set to be equal to $p$ in this step. 
For the sake of completeness, we now give the Riemann solution associated with (3.8). As in [5] and motivated by the ability to treat vacuum zones, we propose taking $a$ nonconstant in each Riemann solution, and we solve

$$
\partial_{t} a+u \partial_{x} a=0 .
$$

The Riemann solutions are given as follows: Let two constant states $\mathcal{V}_{L}=\left(\mathcal{U}_{L},(\rho \Pi)_{L}\right)$ and $\mathcal{V}_{R}=\left(\mathcal{U}_{R},(\rho \Pi)_{R}\right)$ be given, and let $a_{L}$ and $a_{R}$ be two values for $a$. The selfsimilar Riemann solution $(x, t) \mapsto \mathcal{V}\left(x / t ; \mathcal{V}_{L}, \mathcal{V}_{R} ; a_{L}, a_{R}\right)$ associated with (3.8) and initial data

$$
\mathcal{V}(x, t=0)=\left\{\begin{array}{lll}
\mathcal{V}_{L} & \text { if } & x<0 \\
\mathcal{V}_{R} & \text { if } & x>0
\end{array}\right.
$$

is made of four constant states, $\mathcal{V}_{L}, \mathcal{V}_{L}^{*}, \mathcal{V}_{R}^{*}$, and $\mathcal{V}_{R}$, separated by three contact discontinuities associated with $\lambda_{k}=\lambda_{k}(\mathcal{V}), k=1,2,3$, and propagating with speeds denoted by $\lambda\left(\mathcal{V}_{L}, \mathcal{V}_{L}^{*}\right), \lambda\left(\mathcal{V}_{L}^{*}, \mathcal{V}_{R}^{*}\right)$, and $\lambda\left(\mathcal{V}_{R}^{*}, \mathcal{V}_{R}\right)$. More precisely, we have

$$
\mathcal{V}\left(\frac{x}{t} ; \mathcal{V}_{L}, \mathcal{V}_{R}\right)= \begin{cases}\mathcal{V}_{L} & \text { if } \frac{x}{t}<\lambda\left(\mathcal{V}_{L}, \mathcal{V}_{L}^{*}\right), \\ \mathcal{V}_{L}^{*} & \text { if } \lambda\left(\mathcal{V}_{L}, \mathcal{V}_{L}^{*}\right)<\frac{x}{t}<\lambda\left(\mathcal{V}_{L}^{*}, \mathcal{V}_{R}^{*}\right), \\ \mathcal{V}_{R}^{*} & \text { if } \lambda\left(\mathcal{V}_{L}^{*}, \mathcal{V}_{R}^{*}\right)<\frac{x}{t}<\lambda\left(\mathcal{V}_{R}^{*}, \mathcal{V}_{R}\right), \\ \mathcal{V}_{R} & \text { if } \lambda\left(\mathcal{V}_{R}^{*}, \mathcal{V}_{R}\right)<\frac{x}{t}\end{cases}
$$

The intermediate states $\mathcal{V}_{L}^{*}, \mathcal{V}_{R}^{*}$, as well as the speeds of propagation, are determined using for all $k=1,2,3$ the continuity of the (strong) Riemann invariants for $\lambda_{k}$ across the contact discontinuity associated with $\lambda_{l}, l \neq k$. We get after easy calculations $\lambda\left(\mathcal{V}_{L}, \mathcal{V}_{L}^{*}\right)=\lambda_{1}\left(\mathcal{V}_{L}\right)=u_{L}-a_{L} \tau_{L}, \lambda\left(\mathcal{V}_{L}^{*}, \mathcal{V}_{R}^{*}\right)=u^{*}, \lambda\left(\mathcal{V}_{R}^{*}, \mathcal{V}_{R}\right)=\lambda_{3}\left(\mathcal{V}_{R}\right)=u_{R}+a_{R} \tau_{R}$, and

$$
\begin{gathered}
u_{L}^{*}=u_{R}^{*}=u^{*}=\frac{a_{L} u_{L}+a_{R} u_{R}+\Pi_{L}-\Pi_{R}}{a_{L}+a_{R}}, \\
\Pi_{L}^{*}=\Pi_{R}^{*}=\frac{a_{R} \Pi_{L}+a_{L} \Pi_{R}-a_{L} a_{R}\left(u_{R}-u_{L}\right)}{a_{L}+a_{R}}, \\
\frac{1}{\rho_{L}^{*}}=\frac{1}{\rho_{L}}+\frac{a_{R}\left(u_{R}-u_{L}\right)+\Pi_{L}-\Pi_{R}}{a_{L}\left(a_{L}+a_{R}\right)}, \quad \frac{1}{\rho_{R}^{*}}=\frac{1}{\rho_{R}}+\frac{a_{L}\left(u_{R}-u_{L}\right)+\Pi_{R}-\Pi_{L}}{a_{R}\left(a_{L}+a_{R}\right)}, \\
\varepsilon_{L}^{*}=\varepsilon_{L}-\frac{\Pi_{L}^{2}}{2 a_{L}^{2}}+\frac{\Pi^{* 2}}{2 a_{L}^{2}}, \varepsilon_{R}^{*}=\varepsilon_{R}-\frac{\Pi_{R}^{2}}{2 a_{R}^{2}}+\frac{\Pi^{* 2}}{2 a_{R}^{2}} .
\end{gathered}
$$

At this stage, the initial states $\mathcal{V}_{L}$ and $\mathcal{V}_{R}$ and, more precisely, the free parameters $a_{L}$ and $a_{R}$ are implicitly assumed to be such that the waves in the Riemann solutions are ordered as they should be, namely,

$$
\lambda_{1}\left(\mathcal{V}_{L}\right)=u_{L}-\frac{a_{L}}{\rho_{L}}<u^{*}<\lambda_{3}\left(\mathcal{V}_{R}\right)=u_{R}+\frac{a_{R}}{\rho_{R}} .
$$

Following [5], we define $a_{L}=a_{L}\left(\mathcal{V}_{L}\right)$ and $a_{R}=a_{R}\left(\mathcal{V}_{R}\right)$ as follows: if $p_{R} \geq p_{L}$,

$$
\frac{a_{L}}{\rho_{L}}=\max \left(c_{L}, c_{\min }\right)+\alpha\left(\frac{p_{R}-p_{L}}{\rho_{R} c_{R}}+u_{L}-u_{R}\right)_{+},
$$




$$
\frac{a_{R}}{\rho_{R}}=\max \left(c_{R}, c_{\text {min }}\right)+\alpha\left(\frac{p_{L}-p_{R}}{a_{L}}+u_{L}-u_{R}\right)_{+} ;
$$

if $p_{R} \leq p_{L}$,

$$
\begin{aligned}
& \frac{a_{R}}{\rho_{R}}=\max \left(c_{R}, c_{\text {min }}\right)+\alpha\left(\frac{p_{L}-p_{R}}{\rho_{L} c_{L}}+u_{L}-u_{R}\right)_{+}, \\
& \frac{a_{L}}{\rho_{L}}=\max \left(c_{L}, c_{\text {min }}\right)+\alpha\left(\frac{p_{R}-p_{L}}{a_{R}}+u_{L}-u_{R}\right)_{+},
\end{aligned}
$$

with $\alpha=(\gamma+1) / 2, c_{\text {min }}>0$, and where $p_{L, R}=p_{L, R}\left(\mathcal{U}_{L, R}\right), c_{L, R}=c_{L, R}\left(\mathcal{U}_{L, R}\right)$ are the values of the pressures and sound speeds evaluated on $\mathcal{U}_{L}$ and $\mathcal{U}_{R}$.

This choice has several advantages. First, it is shown to fulfill (3.10) and to give the positivity of the intermediate densities $\rho_{L}^{*}$ and $\rho_{R}^{*}$. Then, it complies with the subcharacteristic condition $a>\rho c$. Finally, it guarantees the nonlinear stability of the underlying relaxation scheme that will be described in the next section and the possibility of handling vacuum in the sense that the speeds of propagation $\lambda_{1}\left(\mathcal{V}_{L}\right)$ and $\lambda_{3}\left(\mathcal{V}_{R}\right)$ remain finite. In particular, discrete entropy inequalities as well as maximum principles can be proved. These results are pretty technical to establish and are not recalled in this paper. We refer the reader to [5] for the details.

In the case of PGD, these formulas are to be considered with $p_{L}=p_{R}=0$ and $c_{L}=c_{R}=0$. We then observe that the threshold $c_{\text {min }}$ allows us to guarantee (3.10) when $u_{L} \leq u_{R}$ and then to avoid the resonance phenomenon.

3.3. A relaxation scheme for the gas and pressureless gas dynamics. In this section, we present a relaxation scheme for approximating the solutions of the gas dynamics or PGD equations (3.5) and (3.2) separately. The case of mixed computations involving both the gas dynamics and PGD at the same time will be considered in the next paragraph. It is important to notice that the same formalism will be used for both systems. Just note that in the pressureless case, $E$ must be understood as a function of the unknowns $\rho$ and $\rho u$, namely, $E=(\rho u)^{2} /(2 \rho)$, but not as an unknown with evolution given by the passive transport equation

$$
\partial_{t} \rho E+\partial_{x}(\rho E u)=0
$$

The initial condition is denoted $\mathcal{U}(x, 0)=\mathcal{U}_{0}(x)$, with $E_{0}(x)=\frac{(\rho u)_{0}^{2}(x)}{2 \rho_{0}(x)}$ in the case of PGD. We first set some notation. Let $\Delta x$ and $\Delta t$ be two constant steps for space and time discretizations. Let $\left(x_{j}\right)_{j \in \mathbb{Z}}$ be a sequence of equidistributed points in $\mathbb{R}$ : $x_{j+1}-x_{j}=\Delta x$. For all $j \in \mathbb{Z}$ and all $n \in \mathbb{N}$, we define $x_{j+1 / 2}=x_{j}+\frac{\Delta x}{2}, t^{n}=n \Delta t$, and consider the following discretization of the computational domain $\mathbb{R}_{x} \times \mathbb{R}_{t}^{+}$:

$$
\mathbb{R}_{x} \times \mathbb{R}_{t}^{+}=\bigcup_{j \in \mathbb{Z}} \bigcup_{n \geq 0} C_{j}^{n}
$$

with $C_{j}^{n}=\left[x_{j-1 / 2}, x_{j+1 / 2}\left[\times\left[t^{n}, t^{n+1}[\right.\right.\right.$. On the one hand and as usual in the context of finite volume methods, the approximate solution $\mathcal{U}_{\Delta t, \Delta x}(x, t)$ of (3.5) or (3.2) with initial data $\mathcal{U}_{0}$ is sought as a piecewise constant function on each slab $C_{j}^{n}$ :

$$
\mathcal{U}_{\Delta t, \Delta x}(x, t)=\mathcal{U}_{j}^{n} \quad \text { for }(x, t) \in C_{j}^{n} .
$$


At time $t=0$, we set $\mathcal{U}_{j}^{0}=\frac{1}{\Delta x} \int_{x_{j-1 / 2}}^{x_{j+1 / 2}} \mathcal{U}_{0}(x) d x, j \in \mathbb{Z}$. On the other hand, we define from $\mathcal{U}_{\Delta t, \Delta x}$ the piecewise constant approximate solution $\mathcal{V}_{\Delta t, \Delta x}$ by

$$
\mathcal{V}_{\Delta t, \Delta x}(x, t)=\mathcal{V}_{j}^{n}=\left(\begin{array}{c}
\mathcal{U}_{j}^{n} \\
(\rho \Pi)_{j}^{n}
\end{array}\right) \quad \text { for }(x, t) \in C_{j}^{n} .
$$

This solution is set to be at equilibrium, that is, $(\rho \Pi)_{j}^{n}=p\left(\mathcal{U}_{j}^{n}\right), j \in \mathbb{Z}$, for the gas dynamics and $(\rho \Pi)_{j}^{n}=0, j \in \mathbb{Z}$, for the PGD.

Let us assume that the solution $\mathcal{U}_{\Delta t, \Delta x}\left(x, t^{n}\right)$ at time $t^{n}$ is known. In order to advance it to the next time level $t^{n+1}$, we now describe the two steps of the method in detail.

First step: Evolution in time $\left(t^{n} \rightarrow t^{n+1-}\right)$. In this step, we solve (3.8) with $\mathcal{V}_{\Delta t, \Delta x}\left(x, t^{n}\right)$ as initial data and for times $t \in[0, \Delta t]$. Under the CFL condition

$$
\frac{\Delta t}{\Delta x} \max _{\mathcal{V}}\left(\left|\lambda_{i}(\mathcal{V})\right|, i=1,2,3\right)<\frac{1}{2}
$$

where the maximum is taken over all the $\mathcal{V}$ under consideration, the solution is obtained by solving a sequence of noninteracting Riemann problems set at each cell interface $x_{j+1 / 2}$. It is explicitly known by the previous section, and we have

$$
\begin{gathered}
\mathcal{V}(x, t)=\mathcal{V}\left(\frac{x-x_{j+1 / 2}}{t} ; \mathcal{V}_{j}^{n}, \mathcal{V}_{j+1}^{n} ; a_{L}\left(\mathcal{V}_{j}^{n}\right), a_{R}\left(\mathcal{V}_{j+1}^{n}\right)\right) \\
\left.\left.\quad \text { for }(x, t) \in\left[x_{j}, x_{j+1}\right] \times\right] 0, \Delta t\right], \quad j \in \mathbb{Z} .
\end{gathered}
$$

We then get back a piecewise constant function in $x \in\left[x_{j-1 / 2}, x_{j+1 / 2}\right]$ by means of a classical $L^{2}$ projection, that is,

$$
\left.\left.\tilde{\mathcal{V}}(x, t)=\frac{1}{\Delta x} \int_{x_{j-1 / 2}}^{x_{j+1 / 2}} \mathcal{V}(x, t) d x \quad \text { for }(x, t) \in\left[x_{j-1 / 2}, x_{j+1 / 2}\right] \times\right] 0, \Delta t\right], j \in \mathbb{Z},
$$

and we set

$$
\mathcal{V}_{j}^{n+1-}=\left(\begin{array}{c}
\mathcal{U}_{j}^{n+1-} \\
(\rho \Pi)_{j}^{n+1-}
\end{array}\right)=\tilde{\mathcal{V}}\left(x_{j}, \Delta t\right), \quad j \in \mathbb{Z} .
$$

Of course, this first step is nothing but the celebrated Godunov method applied to (3.8). As a consequence, the updated formula (3.12) can be easily given under the following conservation form:

$$
\mathcal{V}_{j}^{n+1-}=\mathcal{V}_{j}^{n}-\frac{\Delta t}{\Delta x}\left(g\left(\mathcal{V}_{j}^{n}, \mathcal{V}_{j+1}^{n}\right)-g\left(\mathcal{V}_{j-1}^{n}, \mathcal{V}_{j}^{n}\right)\right), \quad j \in \mathbb{Z}, \quad n \geq 0,
$$

where the numerical flux function writes for all $j \in \mathbb{Z}$ as

$$
g\left(\mathcal{V}_{j}^{n}, \mathcal{V}_{j+1}^{n}\right)=\mathcal{G}\left(\mathcal{V}\left(0 ; \mathcal{V}_{j}^{n}, \mathcal{V}_{j+1}^{n} ; a_{L}\left(\mathcal{V}_{j}^{n}\right), a_{R}\left(\mathcal{V}_{j+1}^{n}\right)\right)\right) .
$$

Let us recall that the numerical flux (3.13) is here explicitly known.

Second step: Relaxation $\left(t^{n+1-} \rightarrow t^{n+1}\right)$. We now project the solution $\mathcal{V}_{\Delta t, \Delta x}\left(x, t^{n+1-}\right)$ obtained at the end of the previous step on the equilibrium manifold $\mu=+\infty$. More precisely, we set for all $j \in \mathbb{Z}$

$$
\mathcal{V}_{j}^{n+1}=\left(\begin{array}{c}
\mathcal{U}_{j}^{n+1} \\
(\rho \Pi)_{j}^{n+1}
\end{array}\right)
$$


with

$$
\mathcal{U}_{j}^{n+1}=\mathcal{U}_{j}^{n+1-} \quad \text { and } \quad(\rho \Pi)_{j}^{n+1}=p\left(\mathcal{U}_{j}^{n+1}\right)
$$

in the case of the gas dynamics equations and

$$
\mathcal{U}_{j}^{n+1}=\left(\rho, \rho u, \frac{(\rho u)^{2}}{2 \rho}\right)_{j}^{n+1-} \quad \text { and } \quad(\rho \Pi)_{j}^{n+1}=0
$$

in the pressureless case. This is equivalent to solving in the asymptotic regime, $\mu=$ $+\infty$

$$
\left\{\begin{array}{l}
\partial_{t} \rho=0, \\
\partial_{t}(\rho u)=0, \\
\partial_{t}(\rho E)=0, \\
\partial_{t}(\rho \Pi)=-\mu \rho(p-\Pi)
\end{array}\right.
$$

in the case of the gas dynamics equations and in the case of PGD

$$
\left\{\begin{array}{l}
\partial_{t} \rho=0 \\
\partial_{t}(\rho u)=0 \\
\partial_{t}(\rho E)=-\lambda \rho \varepsilon \\
\partial_{t}(\rho \Pi)=-\mu \rho(p-\Pi) .
\end{array}\right.
$$

In agreement with the description of these two steps, the approximate solution $\mathcal{U}_{\Delta t, \Delta x}$ is then updated according to the following consistent finite volume method:

$$
\begin{gathered}
\rho_{j}^{n+1}=\rho_{j}^{n}-\frac{\Delta t}{\Delta x} \Delta f^{\rho}\left(\mathcal{U}_{j}^{n}, \mathcal{U}_{j+1}^{n}\right), \\
(\rho u)_{j}^{n+1}=(\rho u)_{j}^{n}-\frac{\Delta t}{\Delta x} \Delta f^{\rho u}\left(\mathcal{U}_{j}^{n}, \mathcal{U}_{j+1}^{n}\right),
\end{gathered}
$$

together with

$$
(\rho E)_{j}^{n+1}=(\rho E)_{j}^{n}-\frac{\Delta t}{\Delta x} \Delta f^{\rho E}\left(\mathcal{U}_{j}^{n}, \mathcal{U}_{j+1}^{n}\right)
$$

in the case of gas dynamics and

$$
(\rho E)_{j}^{n+1}=\left(\frac{(\rho u)^{2}}{2 \rho}\right)_{j}^{n+1}
$$

in the case of PGD. Here, of course, $\left(f^{\rho}, f^{\rho u}, f^{\rho E}\right)\left(\mathcal{U}_{j}^{n}, \mathcal{U}_{j+1}^{n}\right)$ denote the first three components of $g\left(\mathcal{V}_{j}^{n}, \mathcal{V}_{j+1}^{n}\right)$ and $\Delta f^{\alpha}\left(\mathcal{U}_{j}^{n}, \mathcal{U}_{j+1}^{n}\right)=f^{\alpha}\left(\mathcal{U}_{j}^{n}, \mathcal{U}_{j+1}^{n}\right)-f^{\alpha}\left(\mathcal{U}_{j-1}^{n}, \mathcal{U}_{j}^{n}\right)$ for $\alpha=\rho, \rho u, \rho E$.

3.4. Coupling the gas and pressureless gas dynamics. In order to perform computations involving both the gas dynamics and PGD at the same time, we have to describe how to couple the relaxation schemes we developed for both systems. Recall that the conservative unknowns are $\rho, \rho u$, and $\rho E$ for the gas dynamics and $\rho$ and $\rho u$ for the PGD. The main difference then clearly lies in the treatment of the energy equation. 
For the sake of clarity, we begin by introducing a color function $Y$ such that $Y=1$ for gas dynamics and $Y=0$ for PGD. From a numerical point of view, a given cell $\mathcal{C}_{j}^{n}$ is said to be pressureless and $Y_{j}^{n}=0$ if the internal energy $\varepsilon_{j}^{n}=\left(\rho E-(\rho u)^{2} / 2 \rho\right)_{j}^{n}$ is less than a given threshold $\varepsilon_{\text {min }}$. Otherwise $\left(\varepsilon_{j}^{n} \geq \varepsilon_{\text {min }}\right)$, the cell is said to be with pressure and $Y_{j}^{n}=1$. Introducing the threshold $\varepsilon_{\min }$ is a convenient way to switch from one algorithm to the other. In agreement with the threshold $c_{\min }$ already introduced for the sound speed in the definition of $a_{L}$ and $a_{R}$, we set

$$
\varepsilon_{\min }=\frac{c_{\min }^{2}}{\gamma(\gamma-1)} \text {. }
$$

Recall indeed that for perfect gas equations of state we have $c^{2}=\gamma(\gamma-1) \varepsilon$. We thus distinguish between zones with PGD where the internal energy is exactly zero and zones where the energy level is above the defined small threshold, a property which is preserved by the pure convective part of the evolution.

Let us consider a given cell $\mathcal{C}_{j}^{n}$. Four different situations must be distinguished, depending in particular on whether $Y_{j-1}^{n}=Y_{j}^{n}=Y_{j+1}^{n}$ or not.

The case $Y_{j-1}^{n}=Y_{j}^{n}=Y_{j+1}^{n}=0$. In this case, we simply use (3.17) and (3.19) without any modification.

The case $Y_{j-1}^{n}=Y_{j}^{n}=Y_{j+1}^{n}=1$. In this case, we simply use (3.17) and (3.18) without any modification.

The case $Y_{j-1}^{n} \neq Y_{j}^{n}$ and/or $Y_{j+1}^{n} \neq Y_{j}^{n}$. In this case, the cell $\mathcal{C}_{j}^{n}$ should be considered with pressure in the update formula. Thus, we propose using

$$
\begin{gathered}
\rho_{j}^{n+1}=\rho_{j}^{n}-\frac{\Delta t}{\Delta x} \Delta f^{\rho}\left(\overline{\mathcal{U}}_{j}^{n}, \overline{\mathcal{U}}_{j+1}^{n}\right), \\
(\rho u)_{j}^{n+1}=(\rho u)_{j}^{n}-\frac{\Delta t}{\Delta x} \Delta f^{\rho u}\left(\overline{\mathcal{U}}_{j}^{n}, \overline{\mathcal{U}}_{j+1}^{n}\right),
\end{gathered}
$$

and

$$
(\rho E)_{j}^{n+1}={\overline{(\rho E)_{j}^{n}}}_{j}^{n}-\frac{\Delta t}{\Delta x} \Delta f^{\rho E}\left(\overline{\mathcal{U}}_{j}^{n}, \overline{\mathcal{U}}_{j+1}^{n}\right),
$$

where, for $k=j-1, j, j+1, \overline{\mathcal{U}}_{k}^{n}=\mathcal{U}_{k}^{n}$ if $Y_{k}^{n}=1$ and

$$
\overline{\mathcal{U}}_{k}^{n}=(\rho, \rho u, \overline{\rho E})_{k}^{n}, \quad \overline{\rho E}_{k}^{n}=\rho_{k}^{n} \varepsilon_{\min }+\left(\frac{(\rho u)^{2}}{2 \rho}\right)_{k}^{n}
$$

otherwise.

3.5. Extension to $2 \mathrm{D}$ configurations and to second-order accuracy. So far, we have focused on the monodimensional case. In order to perform the $2 \mathrm{D}$ computations presented in the next section on Cartesian meshes, we used a very classical dimensional splitting method. We briefly recall that it first consists in splitting the $2 \mathrm{D}$ governing equations into a pair of quasi-1D equations, and then in solving the underlying sequence of two 1D problems with the proposed numerical strategy. Recall that if we denote $(u, v)$ the two components of the velocity field, $v$ being associated with the additional space dimension, the governing equation for $v$ in the quasi-1D system reads

$$
\partial_{t}(\rho v)+\partial_{x}(\rho v u)=0 .
$$


This equation means that $v$ is simply passively transported with the flow. We will thus have to solve the Riemann problem using the same technique as in section 3.2 but with an additional equation:

$$
\left\{\begin{array}{l}
\partial_{t} \rho+\partial_{x}(\rho u)=0 \\
\partial_{t}(\rho u)+\partial_{x}\left(\rho u^{2}+\Pi\right)=0 \\
\partial_{t}(\rho E)+\partial_{x}(\rho E u+\Pi u)=0 \\
\partial_{t}(\rho \Pi)+\partial_{x}\left(\rho \Pi u+a^{2} u\right)=0 \\
\partial_{t}(\rho v)+\partial_{x}(\rho v u)=0
\end{array}\right.
$$

From a numerical point of view, a natural discretization of (3.23), which complies with the exact Riemann solver, is given by

$$
(\rho v)_{j}^{n+1}=(\rho v)_{j}^{n}-\frac{\Delta t}{\Delta x} \Delta f^{\rho v}\left(\mathcal{U}_{j}^{n}, \mathcal{U}_{j+1}^{n}\right),
$$

with $\Delta f^{\rho v}\left(\mathcal{U}_{j}^{n}, \mathcal{U}_{j+1}^{n}\right)=f^{\rho v}\left(\mathcal{U}_{j}^{n}, \mathcal{U}_{j+1}^{n}\right)-f^{\rho v}\left(\mathcal{U}_{j-1}^{n}, \mathcal{U}_{j}^{n}\right)$ and

$$
f^{\rho v}\left(\mathcal{U}_{j}^{n}, \mathcal{U}_{j+1}^{n}\right)=\left\{\begin{array}{ccc}
f^{\rho}\left(\mathcal{U}_{j}^{n}, \mathcal{U}_{j+1}^{n}\right) v_{j}^{n} & \text { if } & f^{\rho}\left(\mathcal{U}_{j}^{n}, \mathcal{U}_{j+1}^{n}\right) \geq 0 \\
f^{\rho}\left(\mathcal{U}_{j}^{n}, \mathcal{U}_{j+1}^{n}\right) v_{j+1}^{n} & \text { if } & f^{\rho}\left(\mathcal{U}_{j}^{n}, \mathcal{U}_{j+1}^{n}\right) \leq 0
\end{array}\right.
$$

This formula was first introduced in [21]. The calculations are left to the reader. The second-order extension in space we used in the numerical experiments is based on a monotonic upstream-centered scheme for conservation laws (MUSCL) reconstruction technique. Regarding the time second-order extension, we used an usual Runge-Kutta method coupled with a Strang splitting. Both are addressed now in more detail, where particular attention is paid to the coupling zones.

MUSCL reconstruction. We begin by briefly recalling the MUSCL method for obtaining the second-order accuracy in space. For more details we refer the reader to the books $[15,37]$ and the original references therein. Consider a change of variables $\mathcal{U} \rightarrow \mathbf{U}=\varphi(\mathcal{U})$. The starting point of the method consists in replacing at each time $t^{n}$ and on each cell $C_{j}$ the constant values $\mathcal{U}_{j}^{n}$ by means of $\varphi$ and a linear reconstruction of $\mathbf{U}$. We set

$$
\left\{\begin{array}{l}
\mathcal{U}^{n}(x)=\varphi^{-1}\left(\mathbf{U}^{n}(x)\right), x \in C_{j}=\left[x_{j-1 / 2} ; x_{j+1 / 2}\right), \text { with } \\
\mathbf{U}^{n}(x)=\mathbf{U}_{j}^{n}+s_{j}^{n} \frac{\left(x-x_{j}\right)}{\Delta x}, \quad \mathbf{U}_{j}^{n}=\varphi\left(\mathcal{U}_{j}^{n}\right), j \in \mathbb{Z} .
\end{array}\right.
$$

In (3.24), $x_{j}$ represents the center of the cell $C_{j}: x_{j}=\frac{1}{2}\left(x_{j-1 / 2}+x_{j+1 / 2}\right)$, and $s_{j}^{n}$ is the slope of the linear reconstruction. The choice of the reconstructed variable $\mathbf{U}$ generally depends on the system under consideration. In the present study, we will perform the reconstruction on the so-called primitive variables $\mathbf{U}=(\rho, u, \varepsilon)$. Note, however, that from now on $\varepsilon$ will be actually reconstructed, i.e., the slope associated with this variable in the linear reconstruction will be possibly nonzero, only if the corresponding cell is with pressure, which means that the color function is such that $Y=1$.

We denote $\mathcal{U}_{j}^{n, \pm}$ and $\mathbf{U}_{j}^{n, \pm}$ the values at the edges $x=x_{j \pm 1 / 2}$ of $\mathcal{U}^{n}$ and $\mathbf{U}^{n}$, respectively:

$$
\left\{\begin{array}{l}
\mathcal{U}_{j}^{n, \pm}=\varphi^{-1}\left(\mathbf{U}_{j}^{n, \pm}\right), \\
\mathbf{U}_{j}^{n, \pm}=\mathbf{U}_{j}^{n} \pm \Delta \mathbf{U}_{j}^{n}
\end{array} \quad \text { with } \quad \Delta \mathbf{U}_{j}^{n}=\frac{1}{2} s_{j}^{n} .\right.
$$

Then, following the basic principle of the MUSCL method, we propose replacing the couple $\left(\mathcal{U}_{j}^{n}, \mathcal{U}_{j+1}^{n}\right)$ with $\left(\mathcal{U}_{j}^{n,+}, \mathcal{U}_{j+1}^{n,-}\right)$ in the evaluation of the numerical fluxes 
$f^{\alpha}\left(\mathcal{U}_{j}^{n}, \mathcal{U}_{j+1}^{n}\right)$ and $f^{\alpha}\left(\overline{\mathcal{U}}_{j}^{n}, \overline{\mathcal{U}}_{j+1}^{n}\right)$ for $\alpha=\rho, \rho u, \rho E$ at each interface $j+1 / 2$ in the previous formulas.

We now draw particular attention to the choice of the reconstructed variable $\mathbf{U}$ and the slopes $s_{j}^{n}$. It is well known that these have to be carefully determined for stability reasons. Once $\mathbf{U}$ is chosen, a usual choice for $s_{j}^{n}$, or equivalently for $\Delta \mathbf{U}_{j}^{n}$, is given by a slope-limiter procedure with, for instance, the so-called minmod limiter. It reads

$$
\Delta \mathbf{U}_{j}^{n}=\frac{1}{2} \operatorname{minmod}\left(\mathbf{U}_{j+1}^{n}-\mathbf{U}_{j}^{n}, \mathbf{U}_{j}^{n}-\mathbf{U}_{j-1}^{n}\right),
$$

where the minmod function is defined by

$$
\operatorname{minmod}(a, b)=\left\{\begin{array}{cc}
\operatorname{sign}(a) \min (|a|,|b|) & \text { if } \\
0 & a b \geq 0,
\end{array}\right.
$$

for two scalar quantities $a$ and $b$ (sign denotes the sign function). In (3.26), minmod is applied component by component. We propose adopting this classical definition whenever $Y_{j}^{n}=1$. When $Y_{j}^{n}=0$, we propose continuing to use this classical definition for the first two components $\rho$ and $u$ of $\mathbf{U}_{j}^{n}$, but setting $\Delta \varepsilon_{j}^{n}=0$ for the last component as already stated above. In other words, the internal energy is not reconstructed when the cell is considered to be without pressure.

Remark. If $Y_{j}^{n}=1$, it may happen that $Y_{k}^{n}=0$ for $k=j-1$ or $k=j+1$. In such a situation $\mathbf{U}_{k}^{n}$ is naturally considered to coincide with $\left(\rho_{k}^{n}, u_{k}^{n}, \varepsilon_{\min }\right)$ in (3.26).

RK2 time integration. In this section, we aim at proposing a time discretization which is second-order accurate in smooth regions and (at least) away from the coupling zones between the pressure and without pressure zones. Of course, the definition of the strategy must take into account the presence of these coupling zones. Actually, our objective is to propose a simple numerical time integration which is equivalent, away from the coupling zones, to the well-known RK2 method (secondorder Runge-Kutta, or Heun) applied to the classical gas dynamics and PGD equations. The reader is assumed to be familiar with this usual RK2 method. Away from the coupling zones, if we use the natural condensed form for the first-order (in time) update formulas

$$
\alpha_{j}^{n+1}=\alpha_{j}^{n}-\frac{\Delta t}{\Delta x} \Delta f^{n, \alpha},
$$

for $\alpha=\rho, \rho u, \rho E$ in the case of gas dynamics and $\alpha=\rho, \rho u$ and

$$
(\rho E)_{j}^{n+1}=\left(\frac{(\rho u)^{2}}{2 \rho}\right)_{j}^{n+1}
$$

in the case of PGD, the RK2 method is made up of two steps which write

$$
\begin{gathered}
\alpha_{j}^{n+1 / 2}=\alpha_{j}^{n}-\frac{\Delta t}{2 \Delta x} \Delta f^{n, \alpha}, \\
\alpha_{j}^{n+1}=\alpha_{j}^{n}-\frac{\Delta t}{\Delta x} \Delta f^{n+1 / 2, \alpha} .
\end{gathered}
$$

As already said, one proposes using these formulas away from the coupling zones, that is to say, whenever $Y_{j-1}^{n}=Y_{j}^{n}=Y_{j+1}^{n}$. In the coupling zones, that is to say, 
whenever $Y_{j}^{n} \neq Y_{j-1}^{n}$ or $Y_{j}^{n} \neq Y_{j+1}^{n}$, the first step (3.29) does not raise any difficulty since it simply consists in applying the previous second-order in space strategy with $\Delta t$ replaced by $\Delta t / 2$ for all $j$. Then, we are able to define the values $Y_{j}^{n+1 / 2}$ for all $j$ depending on whether the values $\varepsilon_{j}^{n+1 / 2}$ are above or below the threshold value $\varepsilon_{\min }$. This is only needed to perform the MUSCL reconstruction as proposed in the previous subsection in order to define the fluxes $f^{n+1 / 2, \alpha}$ in (3.30). Note that the proposed strategy therefore implicitly considers that three fluxes (for mass, momentum, and total energy) are actually needed in (3.30) if $Y_{j-1}^{n}=Y_{j}^{n}=Y_{j+1}^{n}=1$ or in a coupling zone such that $Y_{j}^{n} \neq Y_{j-1}^{n}$ or $Y_{j}^{n} \neq Y_{j+1}^{n}$ in agreement with (3.21) and (3.22). On the contrary, only two fluxes (for mass and momentum) are needed in (3.30) if $Y_{j-1}^{n}=$ $Y_{j}^{n}=Y_{j+1}^{n}=0$. In other words, the values $Y_{j}^{n+1 / 2}$ are only used to perform the reconstructions and calculate the fluxes $f^{n+1 / 2, \alpha}$ and not to determine whether the cell $\mathcal{C}_{j}^{n}$ at time $t^{n}$ has to be considered with or without pressure in (3.30). The values $Y_{j}^{n}$ play this role. This is to ensure a "finite" propagation of a coupling zone during a time step $t^{n} \rightarrow t^{n+1}$.

4. Results and discussion. Let us first underline that the drag and potential source terms are not included in the simulations, except for the 2D Taylor-Green vortices configuration (see the last test cases below). From a numerical point of view, these terms are then applied via operator splitting through an analytical expression of the exponential relaxations. ${ }^{1}$

1D Bouchut-Jin-Li test. To evaluate the performance of the present relaxation scheme in the PGD configuration, the first numerical test of Bouchut, Jin, and $\mathrm{Li}$ [6] (the BJL test) is performed. In this test, the initial solution is designed to create a vacuum state and a mass accumulation. Figure 1 compares the results between the BJL kinetic scheme and the relaxation scheme for first and second orders. Figure 1(a) shows that vacuum is properly captured by the first- and second-order relaxation schemes. As noticed by [6], the first-order scheme forms an artificial density peak. This problem does not appear for either second-order scheme. Compared to the BJL scheme, the second-order relaxation scheme is slightly more diffusive, and the density overshoots created in the zone of negative velocity divergence are a little bit stronger. All the schemes perfectly capture the discontinuity of the velocity in the vacuum region (see Figure 1(b)). In the mass accumulation zone, the most accurate results are obtained with the BJL scheme which is closely followed by the secondorder relaxation scheme. In terms of CPU time, the second-order relaxation scheme costs about twice as much as the single time step BJL scheme because of the two-step Runge-Kutta integration. In return, the relaxation scheme can be extended to accurately solve the Euler equations (see the following test cases) and can be applied on unstructured meshes while the BJL scheme is restricted to PGD and Cartesian grids.

1D Sod shock tube. In order to evaluate the hybrid PGD/gas dynamics relaxation method, the Sod shock tube test is performed with the following initial conditions:

$$
u^{0}=0, \quad\left\{\begin{array}{lll}
\rho^{0}=1, & p^{0}=1.1 & \text { if } \quad x \leq 0.5 \\
\rho^{0}=0.125, & p^{0}=0 & \text { if } \quad x>0.5
\end{array}\right.
$$

\footnotetext{
${ }^{1}$ The exponential relaxation is used here for the particles' drag law, but any form of physical source term can be implemented using operator splitting.
} 
(a)

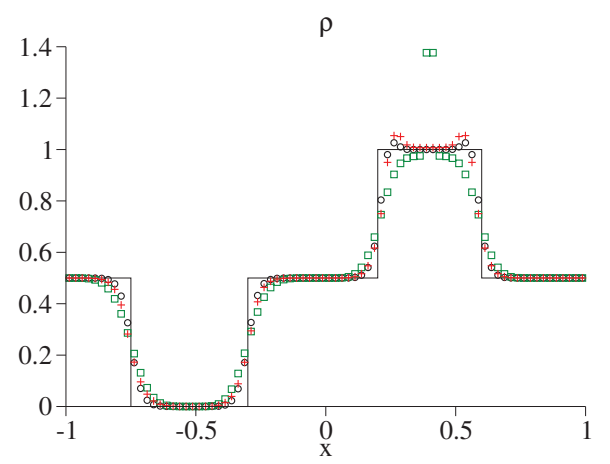

(b)

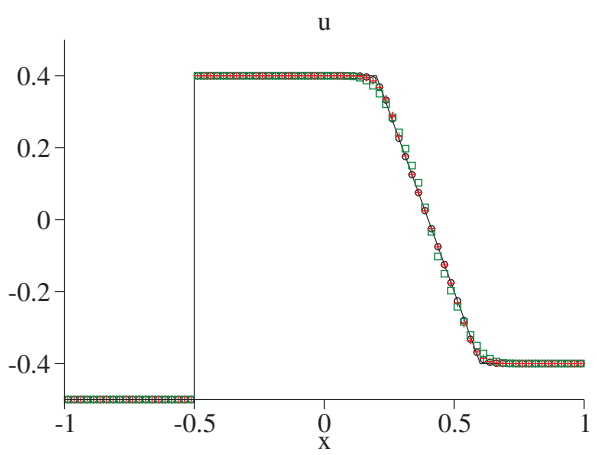

FIG. 1. Profiles of density (a) and velocity (b) for the BJL numerical test at time $t=0.5$ : Exact solution (-), second-order BJL scheme (०), first-order relaxation scheme ( $\square)$, second-order relaxation scheme $(+)$ (80 nodes, $C F L=0.5)$.

At the initial time, $x>0$ corresponds to a zero pressure field computed with the pressureless gas algorithm, while $x \leq 0$ is computed with the gas dynamics algorithm. In this test case, as in all other coupled method calculations, $\varepsilon_{\min }=10^{-10}$ and $c_{\text {min }}$ is evaluated through (3.20) taking $\gamma=1.4$. Figure 2 shows the density and pressure profiles at time $t=0.1644$ for the first- and second-order relaxation schemes. The interface between pressure and pressureless regions does not present any numerical artifact. Due to the poor discretization of the surface discontinuity and the shock, the density solution is smeared by the numerical diffusion. The second-order scheme presents significantly better accuracy than the first-order scheme.

(a)

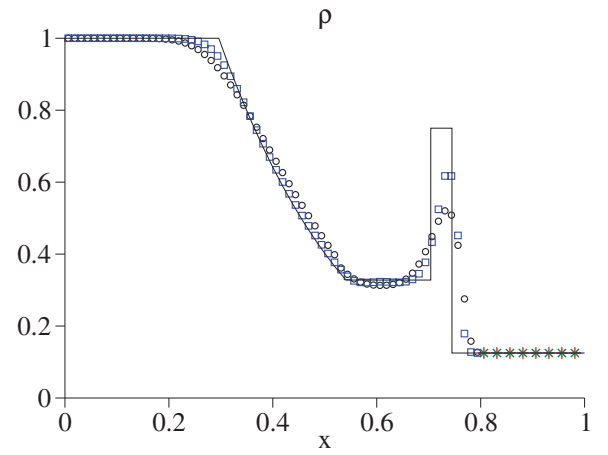

(b)

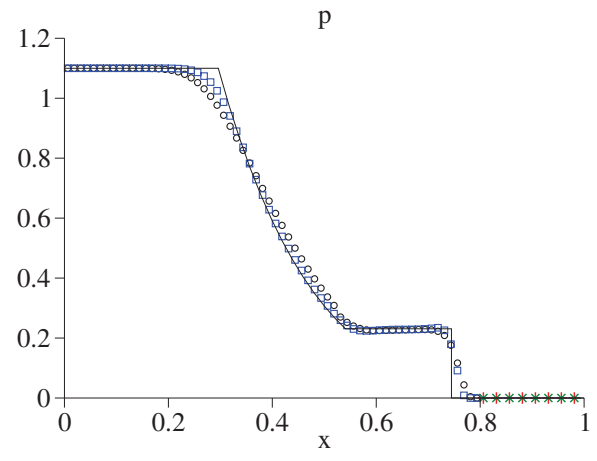

FIG. 2. Profiles of density (a) and pressure (b) for the hybrid relaxation scheme in Sod numerical test at time $t=0.1644$ : first-order relaxation scheme (pressure region: 0 ; pressureless region: + ), second-order relaxation scheme (pressure region: $\square$; pressureless region: $\times$ ) (80 nodes, $C F L=0.5)$.

1D Shock/ $\delta$-shock interaction. The robustness of the second-order relaxation scheme is tested in a configuration where a shock propagates through a pressureless region and meets a $\delta$-shock in density. The $\delta$-shock is created by an initial velocity perturbation located in the pressureless region (see the black line in Figure 3(b)). Figures 3(a) and 3(b) show two instants of the calculation: before $(t=0.1)$ and 
during $(t=0.2)$ the shock/ $\delta$-shock interaction. The trace of this interaction on the velocity profile is an $n$-wave downstream from the shock position (Figures $3(\mathrm{c})$ and $3(\mathrm{~d})$ ). A corresponding density $n$-wave appears in Figure 3(a). After having interacted with the $\delta$-shock $(t=0.4$ and 0.5$)$, the shock has a higher density ratio than before $(t=0.1)$, whereas its velocity jump stays unchanged. This test demonstrates the high robustness of the present relaxation scheme.

(a)

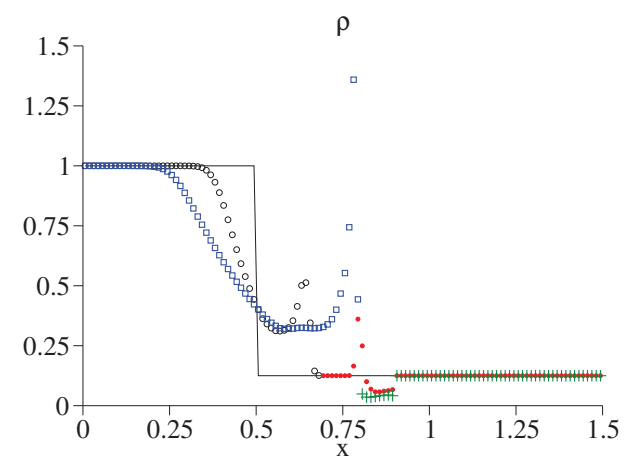

(c)

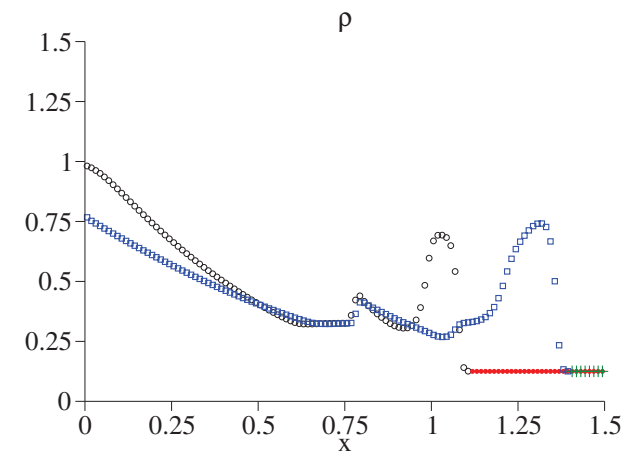

(b)

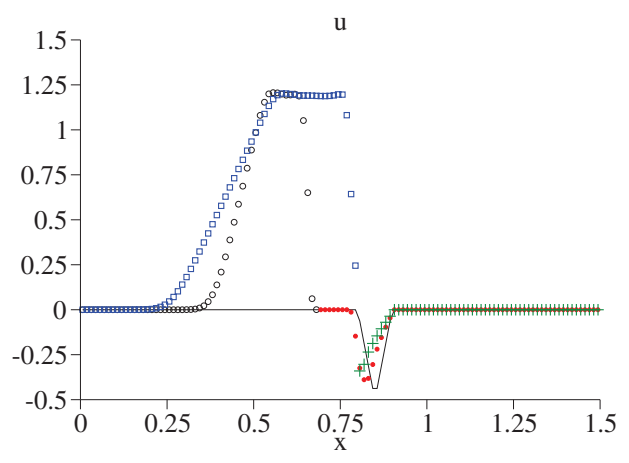

(d)

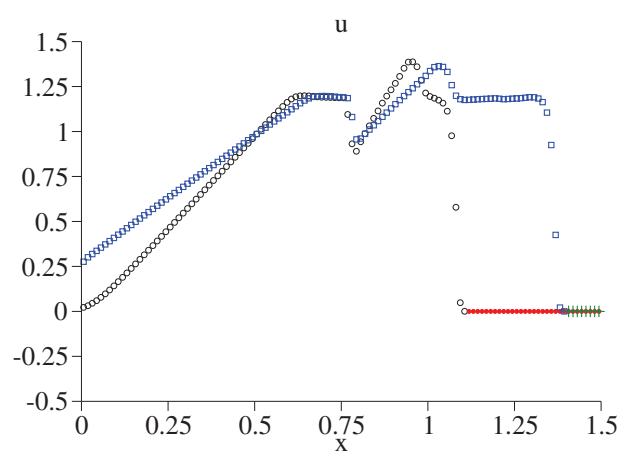

FIG. 3. Second-order hybrid relaxation scheme calculation of the interaction of a shock $/ \delta$ shock: Profiles of density (a), (c) and velocity (b), (d). Before and during the interaction (a) and (b): Time $t=0(-), t=0.1$ (pressure region: $\circ$; pressureless region: $\bullet$ ) and $t=0.2$ (pressure region: $\square$; pressureless region: + ). After the interaction (c) and (d): $t=0.4$ (pressure region: 0 ; pressureless region: •) and $t=0.6$ (pressure region: $\square$; pressureless region: +) (120 nodes, $C F L=0.5$ ).

2D Taylor-Green vortices. Figure $4($ a) shows the velocity field $\mathbf{U}=(U, V)$ of the carrier phase corresponding to the four contrarotating Taylor-Green vortices used in the following numerical tests:

$$
\left\{\begin{array}{l}
U(x, y)=\sin (2 \pi x) \cos (2 \pi y), \\
V(x, y)=-\cos (2 \pi x) \sin (2 \pi y) .
\end{array}\right.
$$

The spray dynamics is coupled to the gaseous flow field through a Stokes drag source term, which amounts to relaxing the spray velocity field toward the gaseous one at a rate set by the Stokes number St, i.e., the nondimensional relaxation time. From [10] we know that there exists a critical value $\mathrm{St}_{c}=1 / 8 \pi$ which separates two regimes. For $\mathrm{St}<\mathrm{St}_{c}$, the particles cannot escape from the Taylor-Green vortices, while, for 
$\mathrm{St} \geq \mathrm{St}_{c}$, they are ejected out of their original vortices. Therefore, the following tests consider two values of $\mathrm{St}$ in order to cover these two regimes: $\mathrm{St}=0.9 \mathrm{St}_{c}$ and $\mathrm{St}=13 \mathrm{St}_{c}$. The initial spray velocity is uniformly zero for all test-cases.

Pressureless transport of a nonuniform initial distribution at supercritical Stokes number. In order to test the method's capability of treating multidimensional transport of inertial particles, the Stokes number is fixed at a supercritical value St $=13 \mathrm{St}_{c}$. Figure 4(a) shows the initial density distribution provided by a cardinal sinus function. To allow comparison with the BJL scheme, the pressureless relaxation scheme is used. Figures 4(b) and 4(c) show the results at time $t=0.8$ for the second-order BJL scheme and the second-order relaxation scheme. Both schemes predict very similar density fields featuring a $\delta$-shock, as expected (see [10]).

(a)

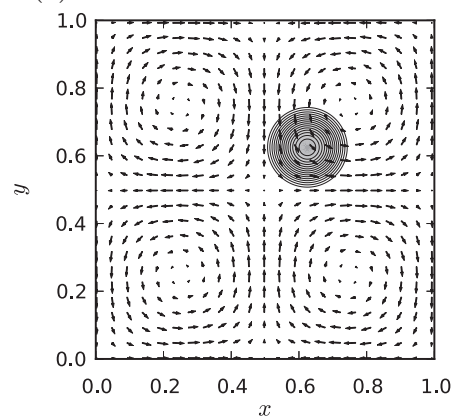

(b)

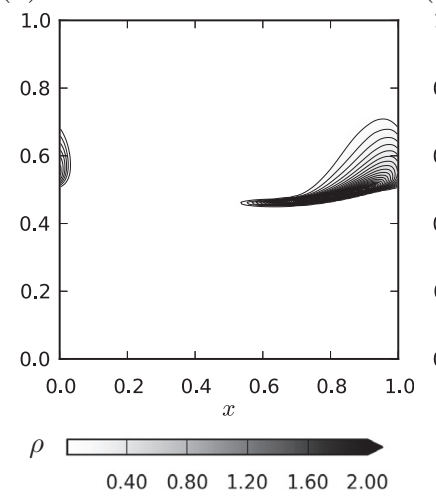

(c)

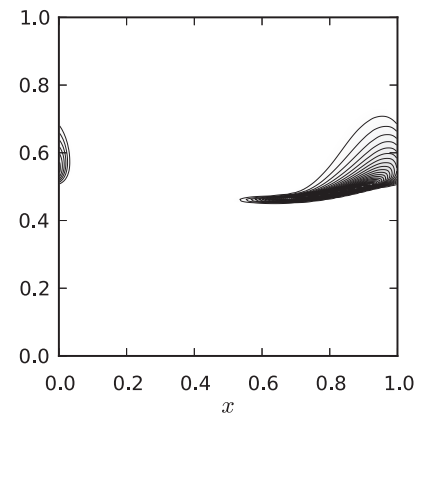

FIG. 4. Carrier phase velocity field (Taylor-Green periodic vortices) and initial density contours (a). Snapshots of the density distribution at time $t=0.8$ for Stokes number $\mathrm{St}=13 \mathrm{St}_{c}$ (200 nodes; $C F L=0.5)$ : Second-order BJL scheme (b) and second-order pressureless relaxation scheme (c).

Pressure versus pressureless transport of a uniform initial distribution at subcritical Stokes number. Figures 5(a) and 5(b) compare the density fields obtained with the second-order BJL scheme and the second-order relaxation scheme, respectively, starting from a uniform density distribution in subcritical Stokes number conditions $\mathrm{St}=0.9 \mathrm{St}_{c}$. As for the supercritical case, both schemes predict very similar results, with mass concentrating in the high strain regions because of the ejection of particles from the center of vortices. ${ }^{2}$ Figure $5(\mathrm{c})$ shows that pressure effects limit the segregation of particles (density concentration here). Physically, this can be interpreted as a mixing effect from the carrier flow turbulence. Here, this turbulence is very simply modeled by a uniform relaxation term of internal energy (target value $\varepsilon_{t}=1$ ).

Hybrid pressure/pressureless transport of a nonuniform initial distribution at supercritical Stokes number. The hybrid scheme is evaluated in a configuration where two parcels of high-inertia particles are ejected from their initial vortices and collide. The density distribution is given by a cardinal sinus function whose center is $(0.125, \pm 0.375)$ and whose radius is 0.125 (see Figure $6 ; t=0$ ). A PGD calculation and a gas dynamics calculation are performed. For the gas dynamics case, a relaxation

\footnotetext{
${ }^{2}$ In the limit of very high Knudsen numbers, the represented solution possesses a singular structure leading to a high concentration of number density of droplets, whereas the Lagrangian solution leads to two streams of droplets crossing without interaction; consequently, such very narrow bands or structures result from the Eulerian modeling assumption of PGD and have nothing to do with the physics of such flows.
} 
(a)

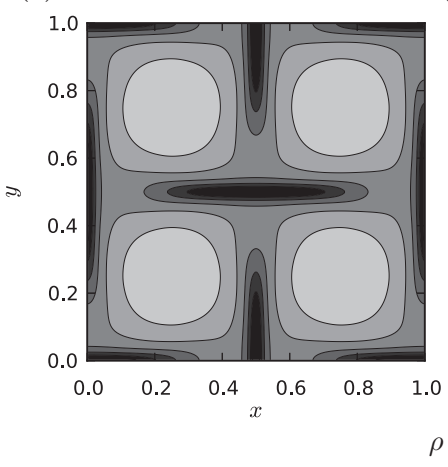

(b)

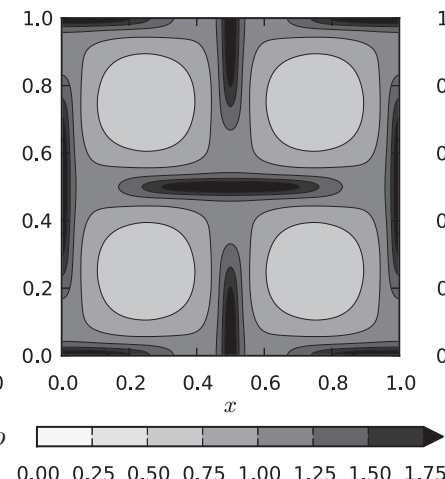

(c)

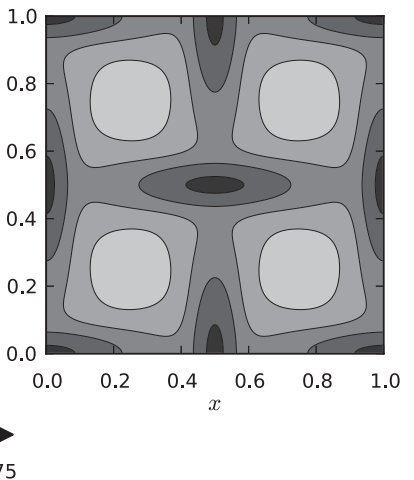

FIG. 5. Snapshots of the density distribution at time $t=0.33$ for Stokes number $\mathrm{St}=0.9 \mathrm{St}_{c}$ : Second-order BJL scheme (a), second-order pressureless relaxation scheme (b), and second-order relaxation scheme with pressure (c) (200 nodes; $C F L=0.5)$.

term of internal energy is imposed using a cardinal sinus function centered on $y=0$ with a radius of 0.125 and a maximum value of $\varepsilon_{t}=0.5$ (see Figure $6 ; t=0 ;$ Hybrid scheme). This energy source term simulates the effect of a local turbulence region of the carrier flow on the particles' transport. As expected, Figure 6 shows that, for both PGD and hybrid schemes, each particle parcel is ejected from its vortex and starts to interact with its mirror image at $t=0.75$. In the PGD case, this interaction forms a $\delta$-shock at the meeting line $y=0$. On the other hand, the hybrid case features only a small increase in density at $y=0$ because pressure effects limit the concentration of particles (see $\rho$ and $p$ figures at $t=0.75$ ). At $t=0.5$, the parcels have just entered the pressure zone, and the pressure effects are negligible. However, at $t=0.75$ the pressure rises rapidly and reaches a maximum value close to $y=0$ where the parcels collide. Later, at $t=1.1$, both schemes evolve differently: most of the density is concentrated close to $y=0$ in the PGD case, while a smoother density distribution and a broader pressure region is observed in the hybrid case.

5. Conclusions. A novel hybrid numerical method for solving Eulerian models for spray dynamics has been proposed. Based on the relaxation method, it can deal with both PGD and general gas dynamics systems of equations in various zones of the same configuration. Therefore, it has the ability, on the one hand, to compute the lowinertia particles dynamics, described by PGD, and, on the other hand, to account for the effects of high-inertia particles in the turbulent regions of the flow, falling under the general gas dynamics framework. The zero-density is also explicitly handled, which is a key feature for simulating spray injection. In terms of accuracy, $1 \mathrm{D}$ and 2D tests in PGD configurations show that the scheme matches the kinetic scheme of Bouchut-Jin-Lin previously used and thus validate the approach. In addition, the hybrid PGD/gas dynamics approach predicts accurate results in the 1D shock tube test-case. The high robustness of the method is demonstrated, particularly in a shock $/ \delta$-shock interaction. $2 \mathrm{D}$ simulations in the framework of Taylor-Green vortices with eventually localized turbulent subgrid energy source allow us to exhibit the potential of the method. Additionally, the relaxation framework makes it possible to handle an arbitrary pressure law such as the real gas-type behavior of turbulent sprays. Therefore, the present investigation shows that this method has the ingredients needed 

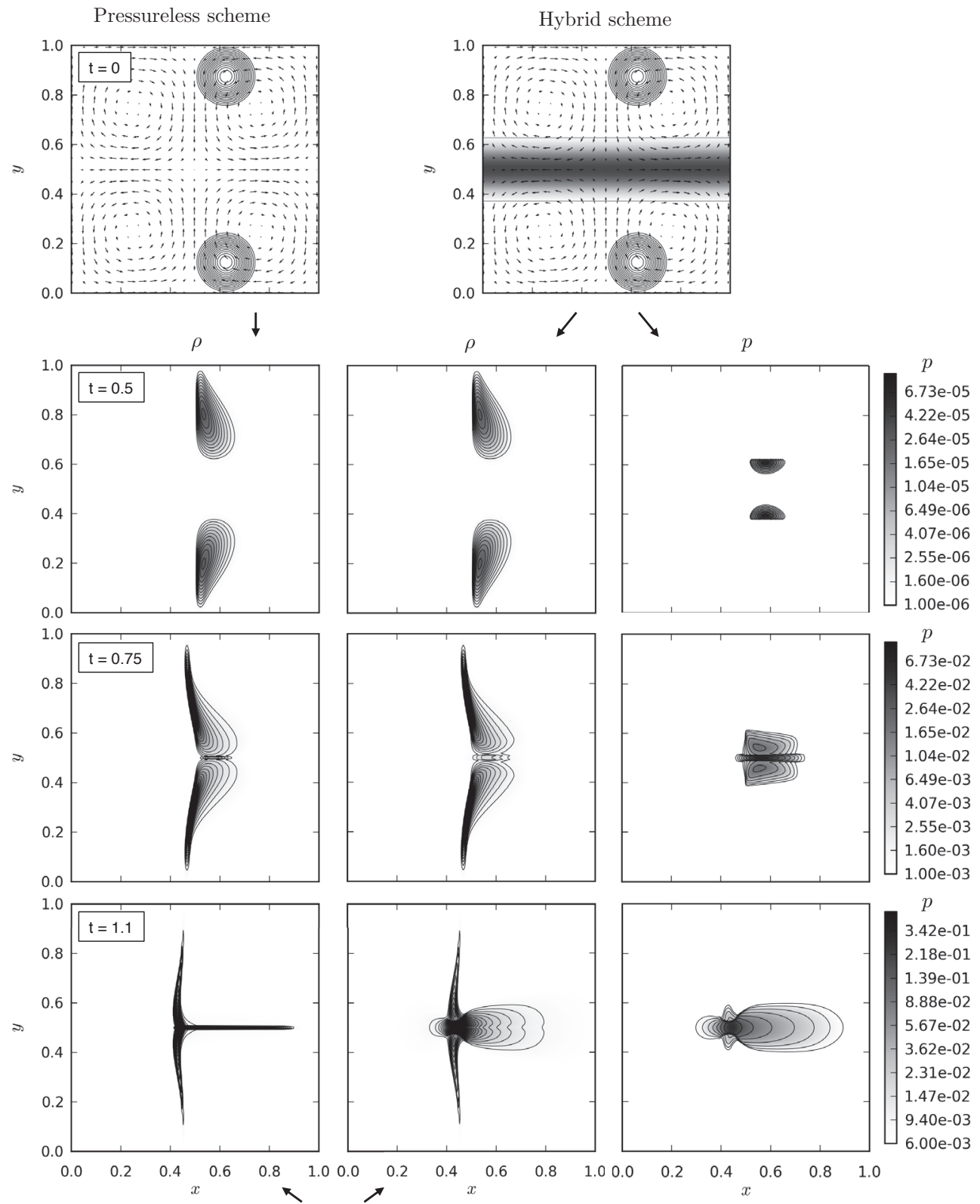

$0.00 \quad 0.250 .500 .751 .001 .251 .50$

FIG. 6. Snapshots of density ( $\rho)$ and pressure ( $p)$ for the second-order pressureless and hybrid schemes at successive times $(t=0.5,0.75$, and 1.1$)$ for $\mathrm{St}=13 \mathrm{St}_{c}(200$ nodes; $C F L=0.5) . t=0$ is the initial density contours and the constant carrier phase velocity field (Taylor-Green periodic vortices). For the hybrid scheme, the constant energy source term is plotted in gray-scale with its upper and lower limits as solid lines. The maximum density at $t=1.1$ is 15.47 for the pressureless scheme and 2.43 for the hybrid scheme.

to simulate turbulent sprays in a DNS/LES framework.

Finally, practical turbulent sprays behave more like a nonequilibrium gas with an 
anisotropic energy distribution. In the future, the present method may be extended to nonisotropic energy distribution by coupling the PGD model in regions of low-inertia particles with nonequilibrium gas dynamics in regions of high-inertia particles using, for example, the Gaussian moment closure proposed by Levermore and Morokoff [23] as in Vié, Doisneau, and Massot [38].

Acknowledgments. Quang Huy Tran and Stéphane de Chaisemartin from IFPEN and Frédérique Laurent from EM2C laboratory are gratefully acknowledged for their useful discussions. The authors also want to thank Cédric Terrier, Jean-François Bourgouin, and Frédéric Cordier for their contributions during their Master student projects at École Centrale Paris.

\section{REFERENCES}

[1] C. Berthon, M. Breuss, And M.O. Titeux, A relaxation scheme for the approximation of the pressureless Euler equations, Numer. Methods Partial Differential Equations, 22 (2006), pp. 484-505.

[2] G. A. Bird, Molecular Gas Dynamics and the Direct Simulation of Gas Flows, Oxford Engrg. Sci. Ser. 42, Oxford University Press, Oxford, UK, 1994.

[3] M. Boileau, S. Pascaud, E. Riber, B. Cuenot, L.Y.M. Gicquel, T. Poinsot, and M. CazaLENS, Investigation of two-fluid methods for large eddy simulation of spray combustion in gas turbines, Flow Turbul. Combust., 80 (2008), pp. 291-321.

[4] M. Bolleau, G. Staffelbach, B. Cuenot, T. Poinsot, and C. Bérat, Les of an ignition sequence in a gas turbine engine, Combust. Flame, 154 (2008), pp. 2-22.

[5] F. Bouchut, Nonlinear stability of finite volume methods for hyperbolic conservation laws and well-balanced schemes for sources, Frontiers in Mathematics, Birkhäuser Verlag, Basel, 2004.

[6] F. Bouchut, S. Jin, And X. Li, Numerical approximations of pressureless and isothermal gas dynamics, SIAM J. Numer. Anal., 41 (2003), pp. 135-158.

[7] C. Chalons and F. Coquel, Navier-Stokes equations with several independent pressure laws and explicit predictor-corrector schemes, Numer. Math., 101 (2005), pp. 451-478.

[8] C. Chalons and J.-F. Coulombel, Relaxation approximation of the Euler equations, J. Math. Anal. Appl., 348 (2008), pp. 872-893.

[9] F. Coquel and B. Perthame, Relaxation of energy and approximate Riemann solvers for general pressure laws in fluid dynamics, SIAM J. Numer. Anal., 35 (1998), pp. 2223-2249.

[10] S. DE Chaisemartin, Modèles Eulériens et simulation numérique de la dispersion turbulente de brouillards qui s'évaporent, Ph.D. thesis, École Centrale Paris, France, 2009; available online from http://tel.archives-ouvertes.fr/tel-00443982/en/.

[11] F. Duchaine, T. Morel, And L.Y.M. Gicquel, Computational-fluid-dynamics-based Kriging optimization tool for aeronautical combustion chambers, AIAA J., 47 (2009), pp. 631-645.

[12] P. Fevrier, O. Simonin, And K. Squires, Partitioning of particle velocities in gas-solid turbulent flows into a continuous field and a spatially uncorrelated random distribution theoretical formalism and numerical study, J. Fluid Mech., 533 (2005), pp. 1-46.

[13] L. Fréret, S. de Chaisemartin, J. Reveillon, F. Laurent, and M. Massot, Eulerian models and three-dimensional numerical simulation of polydisperse sprays, in Proceedings of the 7th International Conference on Multiphase Flow, Tampa, FL, 2010; also available online from http://hal.archives-ouvertes.fr/hal-00498207/en/.

[14] M. Garcia, Development and Validation of the Euler-Lagrange Formulation on a Parallel and Unstructured Solver for Large-Eddy Simulation, Ph.D. thesis, Institut National Polytechnique de Toulouse, Toulouse, France, 2009.

[15] E. Godlewski and P.A. Raviart, Hyperbolic Systems of Conservation Laws, Ellipses, Paris, 1991.

[16] J. B. Greenberg, I. Silverman, and Y. Tambour, On the origin of spray sectional conservation equations, Combust. Flame, 93 (1993), pp. 90-96.

[17] S. Jin AND Z. XIN, The relaxation schemes for systems of conservation laws in arbitrary space dimensions, Comm. Pure Appl. Math., 48 (1995), pp. 235-276.

[18] D. Kah, F. Laurent, L. Fréret, S. de Chaisemartin, R.O. Fox, J. Reveillon, and M. MASsOT, Eulerian quadrature-based moment models for dilute polydisperse evaporating sprays, Flow Turbul. Combust., 85 (2010), pp. 649-676. 
[19] A. Kaufmann, M. Moreau, O. Simonin, and J. Helie, Comparison between Lagrangian and mesoscopic Eulerian modelling approaches for inertial particles suspended in decaying isotropic turbulence, J. Comput. Phys., 227 (2008), pp. 6448-6472.

[20] G. Lacaze, B. Cuenot, T. Poinsot, and M. Oschwald, Large eddy simulation of laser ignition and compressible reacting flow in a rocket-like configuration, Combust. Flame, 156 (2009), pp. 1166-1180.

[21] B. Larrouturou, How to preserve the mass fractions positivity when computing compressible multi-component flows, J. Comput. Phys., 95 (1991), pp. 59-84.

[22] F. Laurent and M. Massot, Multi-fluid modeling of laminar poly-dispersed spray flames: Origin, assumptions and comparison of sectional and sampling methods, Combust. Theory Model., 5 (2001), pp. 537-572.

[23] C. D. LeVermore And W. J. Morokoff, The Gaussian moment closure for gas dynamics, SIAM J. Appl. Math., 59 (1998), pp. 72-96.

[24] M. MAssot, Eulerian multi-fluid models for polydisperse evaporating sprays, in Multiphase Reacting Flows: Modelling and Simulation (Udine, 2006), D. L. Marchisio and R. O. Fox, eds., CISM Courses and Lectures 492, Springer, Wien, New York, Vienna, 2007, pp. 79-123.

[25] M. Massot, S. De Chaisemartin, L. Fréret, D. Kah, and F. Laurent, Eulerian multi-fluid models: Modeling and numerical methods, in Modeling and Computations of Nanoparticles in Fluid Flows, Lecture Series of the von Karman Institute, NATO-RTO, Neuilly sur Seine, France, 2009, pp. 1-86.

[26] M. Massot, R. Knikker, C. PÉra, And J. Reveillon, Lagrangian/Eulerian analysis of the dispersion of evaporating sprays in non-homogeneous turbulent flows, in Proceedings of the 5th International Conference on Multiphase Flow, Yokohama, Japan, 2004, paper 262.

[27] M. Moreau, B. BÉdat, and O. Simonin, A priori testing of subgrid stress models for EulerEeuler two-phase LES from Euler-Lagrange simulations of gas-particle turbulent flow, in 18th Annual Conference on Liquid Atomization and Spray Systems, ILASS Americas, Irvine, CA, 2005.

[28] P. J. O'Rourke, Collective drop effects on vaporizing liquid sprays, Ph.D. thesis, Princeton University, Princeton, NJ, 1981.

[29] R.V.R. PANDyA And F. MASHAYEK, Two-fluid large-eddy simulation approach for particle-laden turbulent flows, Internat. J. Heat Mass Transfer, 45 (2002), pp. 4753-4759.

[30] M.W. REEKs, On the continuum equations for dispersed particles in nonuniform flows, Phys. Fluids A, 4 (1992), pp. 1290-1303.

[31] E. Riber, M. Moreau, O. Simonin, and B. Cuenot, Towards large eddy simulation of nonhomogeneous particle laden turbulent gas flows using Euler-Euler approach, in 11th Workshop on Two-Phase Flow Predictions, Merseburg, Germany, 2005.

[32] E. Riber, V. Moureau, M. García, T. Poinsot, and O. Simonin, Evaluation of numerical strategies for large eddy simulation of particulate two-phase recirculating flows, J. Comput. Phys., 228 (2009), pp. 539-564.

[33] A. Roux, L.Y.M. Gicquel, Y. Sommerer, And T. Poinsot, Large eddy simulation of mean and oscillating flow in side-dump ramjet combustor, Combust. Flame, 152 (2008), pp. 154176.

[34] L. Selle, L. Benoit, T. Poinsot, F. Nicoud, And W. Krebs, Joint use of compressible largeeddy simulation and Helmholtz solvers for the analysis of rotating modes in an industrial swirled burner, Combust. Flame, 145 (2006), pp. 194-205.

[35] O. Simonin, P. FÉvrier, And J. Laviéville, On the spatial distribution of heavy-particle velocities in turbulent flow: From continuous field to particulate chaos, J. Turbul., 3 (2002), pp. $1-18$.

[36] I. Suliciu, Energy estimates in rate-type thermo-viscoplasticity, Internat. J. Plast., 14 (1998), pp. $227-244$.

[37] E. Toro, Riemann Solvers and Numerical Methods for Fluid Dynamics: A Practical Introduction, Springer-Verlag, Paris, 1999.

[38] A. Vié, F. Doisneau, And M. Massot, On the anisotropic Gaussian velocity closure for inertial- particle laden flows, Commun. Comput. Phys., 17 (2015), pp. 1-46; also available online from http://hal.archives-ouvertes.fr/hal-00912319.

[39] A. Vié, S. Jay, B. Cuenot, And M. Massot, Accounting for polydispersion in the Eulerian large eddy simulation of the two-phase flow in an aeronautical-type burner, Flow Turbul. Combust., 90 (2013), pp. 545-581.

[40] A. Vié, M. Sanjosé, S. Jay, C. Angelberger, B. Cuenot, and M. Massot, Evaluation of a multifluid mesoscopic Eulerian formalism on the large eddy simulation of an aeronauticaltype configuration, in Proceedings of the 7th International Conference on Multiphase Flow, Tampa, FL, 2010, pp. 1-11. 
[41] F. A. Williams, Spray combustion and atomization, Phys. Fluids, 1 (1958), pp. 541-545.

[42] L.I. ZAICHIK, A statistical model of particle transport and heat transfer in turbulent shear flows, Phys. Fluids, 11 (1999), p. 1521.

[43] L.I. Zaichik, O. Simonin, And V.M. Alipchenkov, An Eulerian approach for large eddy simulation of particle transport in turbulent flows, J. Turbul., 10 (2009), pp. 1-21. 